\title{
The MASE design experience
}

\author{
$\underline{\text { C. G. Ralha }}^{\text {a }}{ }^{\text {, C. G. Abreu }}{ }^{\text {a }}$, C. G. C. Coelho ${ }^{\mathrm{a}}$ and Bruno Macchiavello ${ }^{\mathrm{a}}$ \\ ${ }^{a}$ Computer Science Department, Brasília University, PO BOX 4466, Zip Code 70.904-970, Brasília, Brazil \\ Email: ghedini@cic.unb.br
}

\begin{abstract}
In Computer science, agent-based modeling (ABM) and multi-agent simulation contribute to scenario anal-ysis, since they may represent patterns of human interaction, which are essential to a better understanding of real systems, including many different aspects such as social, economical and environmental. This paper presents the use of agent-based techniques to define and develop the MASE Multi-Agent System for Envi-ronmental simulation model using two different design approaches: the TROPOS Agent Oriented Software Engineering methodology with JADE framework, and the Agent-ObjectRelationship modeling and simulation platform. MASE model system was defined to characterize land-use change dynamics through a replicable pa-rameterization process, useful to the development of agent-based simulation frameworks. However, different agent-based design experiences can enrich the modeling and development process of environmental simulation frameworks, since they embed conceptual structure definition according to specific design concepts. Thus, in this paper we include a short discussion of challenges, perspectives and limitations of two design experiences involving different concepts and techniques for information systems analysis. We believe the reported discus-sion can serve as a starting point to define evaluation criteria for comparative analysis of different agent-based design approaches. To illustrate land-use simulations with MASE we used the Brazilian Cerrado case study.
\end{abstract}

Keywords: Agent-based modeling, design options, process design, brazilian cerrado case 


\section{INTRODUCTION}

The natural and engineering sciences have enormously progressed with the advent of computers. Computers could make a contribution to a better understanding of social and economic systems being relevant also for the statistical analysis of data and data-driven efforts to reveal patterns of human interaction. In Computer Science, multi-agent simulation (MAS) can be seen as experimental technique for hypothesis testing and scenario analysis, which can be used complementary and in combination with experiments in real-life, the lab or the Web (Parker et al., 2003; Helbing, 2012). A complementary method that seems to be suited for the computer simulation of socio-economic systems is the agent-based modeling (ABM) (Bonabeau, 2002). The use of ABM and MAS contribute to scenario analysis, since they may represent patterns of human interaction which are essential to a better understanding of real systems, including many different aspects such as social, economical and environmental.

Thus, agent-based computational models appear ideal to study interdependencies between different human activities assuming symbiotic or competitive relationships, considering social and economic systems from an ecological perspective (May and McLean, 2007). Agents are very powerful and flexible modeling entities that depending on the problem of interest may represent individuals, groups, companies, government, countries, continents, with specific behaviors and interactions that may be formalized by equations or logical operations such as decision rules. Such behavioral rules consider individual variations maintaining the heterogeneity aspects and random influences or variations considering stochasticity of complex real models.

The use of computational agents is focussed in this paper by describing the design experience of MASE Multi-Agent System for Environmental simulation (Ralha et al., 2013). MASE was developed as a multiagent model system to characterize land-use change dynamics. MASE was previously compared in relation to the least cost pathway model (widely used application of GIS) to non agent-based environmental frameworks (e.g., Dinamica EGO, ArcGIS, IDRISI) (Abreu et al., 2012). MASE model adopts a replicable parameterization process useful to the development of simulation frameworks based on a two-fold methodological approach: (i) the systematic and structured empirical characterization model of Smajgl et al. (2011); and (ii) the conceptual structure definition according to the agent-based model documentation protocol - Overview, Design concepts and Details (ODD) (Grimm et al., 2006, 2010). The MASE technical details, implementation aspects, illustration of simulation with the Brazilian Cerrado case using real LANDSAT ETM images and the model validation are available in Ralha et al. (2013).

In order to compare quantitative and qualitative aspects of the MASE model we applied two scientifically rigorous statistical techniques of map comparison to land change models. The methods were developed by Pontius et al. (2008) to compare: a reference map of initial time, a reference map of subsequent time, and a prediction map of the subsequent time. According to the authors, the three possible two-map comparisons for each application characterize: the dynamics of the landscape, the behavior of the model, and the accuracy of the prediction. The authors also propose the three-map comparison for each application to specify the amount of the predictions accuracy that is attributable to land persistence versus land change. According to the method the applications can be summarized and compared using two statistics: the null resolution and the figure of merit. MASE results considering Pontius et al. (2008) method with the Cerrado case presented good simulation accuracy, since the application results were better than the null model (what examines both the behavior of the model and the dynamics of the landscape) and the figure of merit was greater than $50 \%$ (what means that the amount of correctly predicted change is larger than the sum of any type of error).

In this paper, MASE is going to be described by two different design approaches that involve particular concepts and techniques for information systems analysis. The rest of the article includes in Section 2 the MASE design using TROPOS Agent Oriented Software Engineering (AOSE) methodology. Section 3 introduces the MASE design according to the ABM and simulation paradigm using the Agent-Object-Relationship (AOR) model. Section 4 presents the Brazilian Cerrado case study to illustrate both design experiences presented in Sections 2 and 3. Finally, in Section 5, we conclude the article discussing both design approaches in respect of the challenges, perspectives, limitations and points some future work.

\section{MASE: AOSE DESIGN}

The AOSE approach can adopt different methodologies among many proposals: Gaia (Wooldridge et al., 2000), MaSE (Deloach, 2001; DeLoach et al., 2001), Prometheus (Padgham and Winikoff, 2002a,b) and TROPOS (Giunchiglia et al., 2002; Bresciani et al., 2004; Giorgini et al., 2004). In this paper, the MASE AOSE design adopted TROPOS, which is based on the Object-Oriented (OO) approach using the Unified Modeling 


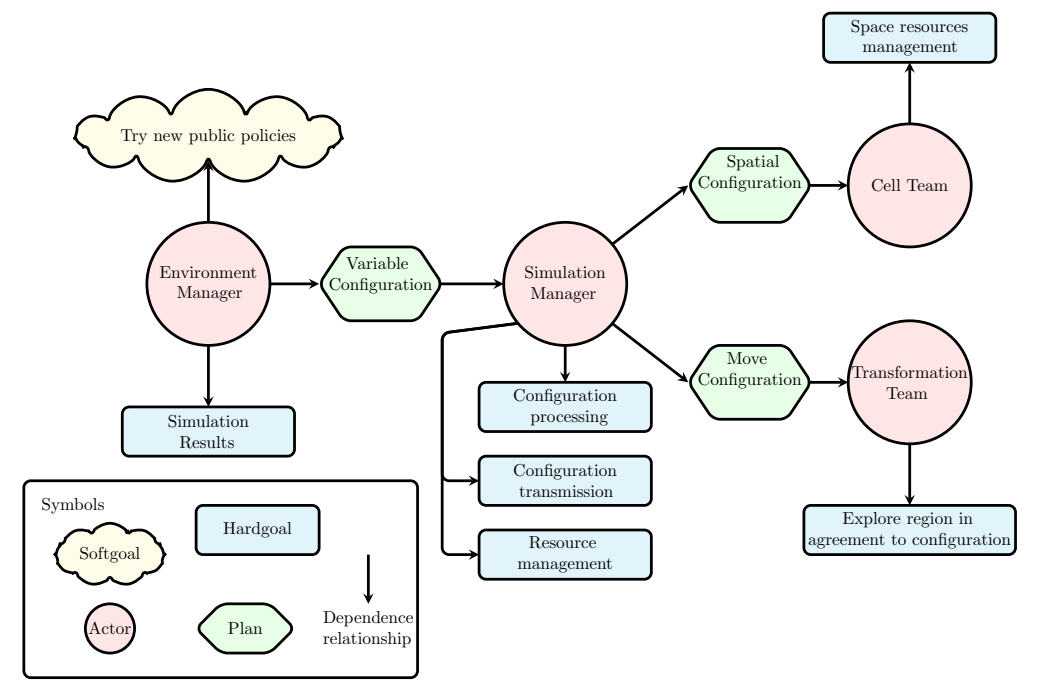

Figure 1. MASE early requirements using TROPOS methodology (agents, goals, plans)

Language - UML (Rumbaugh et al., 2004) and the Agent UML - AUML (Padgham and Winikoff, 2003). According to Bresciani et al. (2004), the TROPOS methodology is intended to support all analysis and design activities in the software development process. TROPOS rests on the idea of building a system model that is incrementally refined and extended, providing a common interface to various software development activities, as well as a basis for documentation and evolution of the software. TROPOS is based on two key ideas: (i) the notion of agent and all related mentalistic notions (goals and plans) are used in all phases of software development, from early analysis down to the actual implementation; and (ii) it covers also the very early phases of requirements analysis, thus allowing for a deeper understanding of the environment.

Figure 1 presents the TROPOS diagram of early requirements analysis that includes: one softgoal (try new public policies), six hardgoals (e.g. simulation results, space resources management) which are related to the four actors (environment manager, simulation manager, cell team, transformation team), three plans (variable configuration, spatial and move config) and the dependence relationship (represented by the arrows). The diagram presents the relationship among the environment manager, the simulation manager and the agent teams (cell and transformation) through the different configuration (config) plans, since the spatial and movement representation in the simulation grid is parameterized using configuration variables.

MASE was modeled using TROPOS with a prototype implemented in the middleware JADE-Java Agent Development Framework, version 4.0, released on 20 April, 2010 (Bellifemine et al., 2007). JADE is written in Java language for developing and executing applications based on intelligent agents being compliant to FIPAFoundation for Intelligent Physical Agents specifications. To manipulate the images we used the open source ImageJ Lybrary. As input images for the simulation process we used already classified raster-GIS program images obtained by satellite monitoring LANDSAT EMT, being classified by the PROBIO software (Brazilian Institute of Environment and Renewable Natural Resources (IBAMA)/Environment Ministry (MMA), Remote Sensing Center (CSR)-IBAMA/MMA). MASE is a software developed in the University of Brasília, registered by number BR 5120130005280 at the National Industrial Property Institute - Federal District of Brazil (INPI/DF), being available for academic purposes (https: // sourceforge. net/p/mase-unb).

\section{MASE: AOR DESIGN}

The AOR approach introduced by Wagner (2004) puts together the ABM and MAS as complementary methods. Although there are several agent-based simulation platforms (Railsback et al., 2006), we turned our attention to the AOR platform, since it is based on a high-level declarative specification language the AOR Simulation Language (AORSL). The AORSL has a UML-based visual syntax and an underlying simulation metamodel, as well as an abstract simulator architecture and an execution model. This approach is an extension and refinement of the classical discrete event simulation paradigm by enriching it with the basic concepts of the AOR metamodel (Wagner, 2003). The AORSL has special constructs to represent agent cognitive as- 


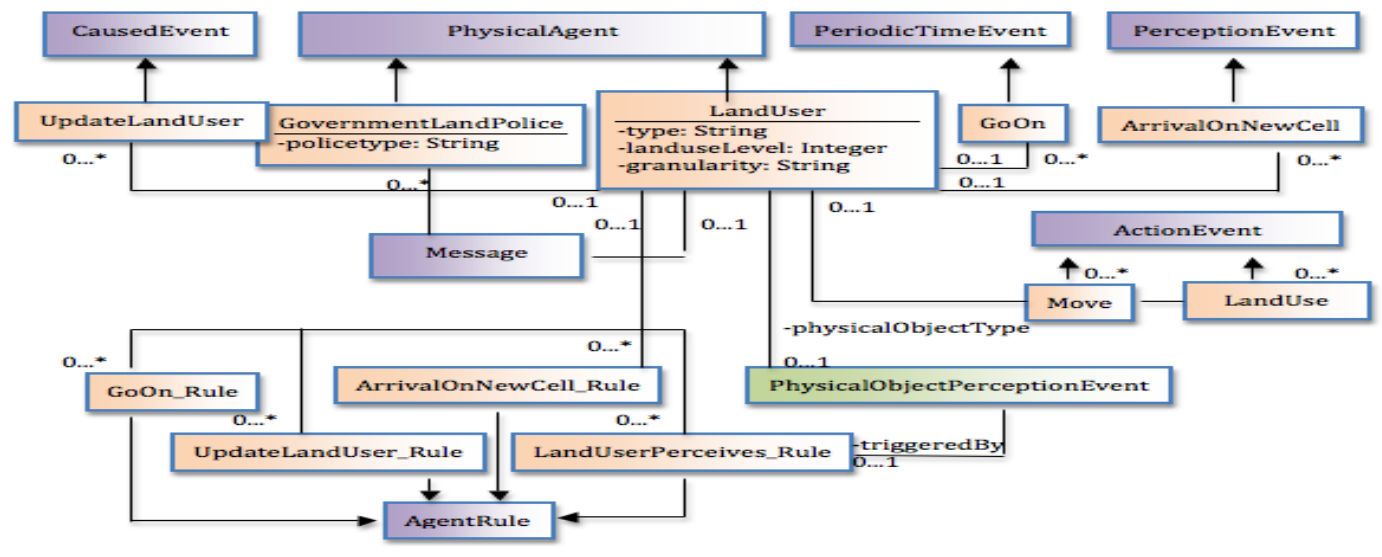

Figure 2. MASE model using AOR simulation model

pects based on their beliefs and intentions (Wagner and Diaconescu, 2009). All of these constructs (i.e., entity types, agent rules, environment rules, and initial conditions) are specified using the AORSL, an XML-based language that allows embedding Java code in order to implement auxiliary functions and expressions. In fact, the scenario specification in AORSL is transformed into Java code by the AOR system. Running the simulation amounts to compiling and running the auto-generated Java code.

The AOR is a discrete event-oriented simulation platform, where agents respond to events in their environment by executing actions and interacting with each other. Basically, there are two different kinds of events: (i) an exogenous event, that is an external event (such as the arrival of a new customer) which does not depend on the actions of agents; and (ii) an internal event, like a message exchange, that is the basis of simulation since agents interact sending messages to one another. Usually, the occurrence of an exogenous event is what triggers a simulation run. To run multiple instances of a process, the AOR system schedules the occurrence of exogenous events to trigger the whole process at different simulation time.

Figure 2 presents the specification of MASE scenario using AOR model with the necessary entity types, which include the agent types, messages and events used in the simulation scenario. The behavior of agents is specified by means of agent rules. Typically, an agent rule defines that when a certain message is received, another message is produced and sent to other agents. Since the rules for each agent are defined separately the simulation scenario is effectively implemented in a decentralized way by the combined behavior of agents. Another kind of rules that exist in the AOR system are environment rules. Basically, these have to do with the occurrence of exogenous events and they define what should be done when such events occur. An environment rule specifies that when a certain event occurs, a message should be sent to some agent. The sending of this message triggers a rule of the receiving agent, which creates a chain of message exchanges that puts the whole simulation in motion. Environment rules also have the ability to create and destroy agents. A set of initial conditions for the simulation scenario specifies which agents already exist at the beginning of the simulation. The initial conditions also include a schedule for the occurrence of at least one exogenous event to trigger the simulation. Since the environmental land-use simulation need to deal with real maps (e.g. possible satellite images), there is the necessity to import from raster-GIS image files the spacial elements to the simulation grid. Unfortunately, there is a grid size limitation in the AOR platform experimentally tested as $50 \times 50$ for the axes X/Y (http://www.simurena.de/en/simurena-framework_en).

\section{CASE STUDY}

To illustrate the use of MASE design experiences with AOSE (Section 2) and AOR (Section 3), we used the Federal District Cerrado case. The Brazilian Cerrado is the major Brazilian savanna-like ecosystem and the second largest biome in South America (Oliveira, 2002; Klink and Moreira, 2002). The Cerrado savanna of central Brazil has been undergoing rapid transformation to cattle ranching and more recently to soybeans (soya) production, requiring urgent action for the conservation of biological richness in the face of threats of destruction (McAlpine et al., 2009). According to a recent report released by WWF UK, the increased use of soya has had painful consequences for the Cerrado region of Brazil. 


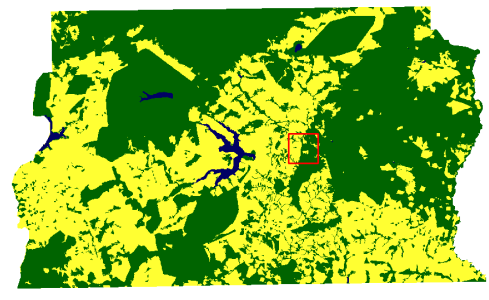

(a)

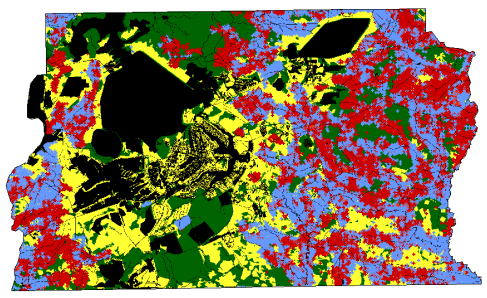

(b)

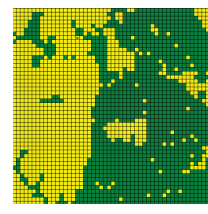

(c)

Figure 3. Federal District images: (a) JADE framework-2002; (b) JADE 2008; (c) AOR platform-2002

The report states that in the six year period from 2002 to 2008, land-use change in the Cerrado released 275 million tons of $\mathrm{CO} 2$ per year, more than half the total emissions for the United Kingdom. For the cited reasons, the Federal District was selected for our case study, since it is the single state of Brazil fully covered by the Cerrado biome (http://blogs. worldwatch.org/nourishingtheplanet/ wwf-report-soya-and-the-cerrado-brazil's-forgotten-jewel/).

The Federal District area of $5,789 \mathrm{~km}^{2}$ is divided into cells of the simulation grid using two different maps to compare from a initial time (2002-t0) to a subsequent time (2008-t6). Each time-step in our simulation corresponds to a week of real time, resulting in 365 steps to cover the length of years. Every set of four cells represents one hectare, which is occupied by a different land-user agent. The physical state of the cells correspond to the set of real spatial data including six proximal variables: water courses (rivers); water bodies (lakes); buildings; highways; streets; and protected areas. Figure 3 presents Federal District LANDSAT ETM images representing 2002 (a,c) and 2008 (b) used with JADE framework (a, b) and AOR platform (c).

In Figure 3 the yellow part represents the anthropic used land, the green the native vegetation and the dark blue the water courses. In Figure 3 (b) the six proximal variables are colored in black, and there are two different land-user agents: farmers (blue) and ranchers (red). Note in the upper right quadrant of (b), where rancher and farmer agents are using the land, there is an enormous land-use change compared to the green native vegetation of (a). Figure 3 (c) represents the 2002 image used as input in AOR platform, which is marked in red in (a). This small part is a $50 \times 50$ grid, since the total area of the Federal District has a grid size of $940 \times 644$ not feasible to AOR platform due to processing limitations. Note that the grid size of (c) represents $0,4 \%$ of the total Federal District grid area and we didn't simulate the same land-use change using the AOR platform since we would need to divide the real image in 250 pieces and simulate each one in separate.

\section{DISCUSSION AND CONCLUSIONS}

This paper presented research using two different design approaches with MASE. This study had the objective to analyze the conceptual structure definition according to specific design concepts and details in order to help the modeling and development process of environmental simulation frameworks. Thus, the challenges, perspectives and limitations of the approaches presented have to be evaluated by simulation scenario developers, since they embed different concepts and techniques. The first approach presented in Section 2 uses an AOSE orientation with TROPOS methodology and JADE framework. The second approach of Section 3 uses an $\mathrm{ABM}$ and simulation paradigm with AOR platform. The main challenge we faced with both approaches is related to the modeling design aspects and implementation characteristics. The TROPOS AOSE methodology includes all phases of software development, from early analysis down to the implementation, building a model of the system-to-be and its environment early phases of requirements analysis (Figure 1). The AOSE approach allows a deeper understanding of the environment, where the software must operate and of the interactions that should occur between software and agents. The focus of this approach is the agents definition, their goals, the communication and interaction protocols and the plans to achieve the desired results through the agent behaviors.

The AOR simulation metamodel is based on a discrete event paradigm that integrates the concepts of AgentObject-Relationship into the AORSL. To define a simulation scenario, the high-level declarative specification XML-based language has to be learned. The simulation metamodel definition have to identify all entities 
involved in the simulation scenario (Figure 2), including physical objects and agents, atomic events with internal events (periodic time and reminder event) and environment events (caused, action, exogeneous events), and the messages to be exchanged among agents. After the metamodel definition, the simulation has to be coded in AORLS language to be executed in the AOR platform. Unfortunately, the use of real raster-GIS images impose a strong limitation in terms of grid space size. Specially considering MASE simulations that use images obtained by satellite monitoring (Figure 3 ). Thus, image manipulation to divide the Federal District area $\left(5,789 \mathrm{~km}^{2}\right)$ must be proceeded and the script to import all the image elements to the AOR simulation grid is necessary, before the simulation can be started (simulation initial state). Despite the modeling expressiveness of the AORSL, the use of AOR platform demands an extra effort to deal with real images, what can complicate the land-use simulation process considering different resource constraints.

As future work, we intend to improve agents rationality to emulate human reasoning, from simple finite state machines and rule based agent behaviors to sophisticated Belief-Desire-Intention (BDI) model, which was defined by Bratman (1987) for agents cognition, considering limited processing resources. We consider this is the most challenging aspect of the research focussing the multiple scale integration of ABM and MAS for complex environmental systems. In this direction, it is important to consider the complexity in different levels (macro and micro), specially when studying social phenomena where modeling complex entities as individuals or particles is not enough. Nevertheless, we believe the research carried out with MASE different design experiences, may help to define a very comprehensive rational model to agents-based simulations involving multiple scales based on the practical reasoning systems paradigm.

\section{ACKNOWLEDGeMents}

The first author would like to thank the financial support from the Technological and Scientific Ventures Foundation (Fundação de Empreendimentos Científicos e Tecnológicos-FINATEC) - Edital 03/2013 de participação em eventos científicos internacionais.

\section{REFERENCES}

Abreu, C. G., C. G. Coelho, C. G. Ralha, A. Zaghetto, and B. Macchiavello (2012). Comparison of the mase system to different computational frameworks using a least cost pathway model. In Proceedings of the 8th international Conference on Ecological Informatics - ISEI'13.

Bellifemine, F. L., G. Caire, and D. Greenwood (2007). Developing Multi-Agent Systems with JADE. John Wiley \& Sons.

Bonabeau, E. (2002). Agent-based modeling: Methods and techniques for simulating human systems. Proc. of the National Academy of Sciences of the United States of America 99(Suppl 3), 7280-7287.

Bratman, M. (1987). Intention, plans, and practical reason. Cambridge, MA: Harvard University Press.

Bresciani, P., P. Giorgini, F. Giunchiglia, J. Mylopoulos, and A. Perini (2004, May). TROPOS: An agentoriented software development methodology. Autonomous Agents and Multi-Agent Systems 8(3), $203-236$.

Deloach, S. A. (2001). Analysis and design using mase and agenttool. In Proceedings of the 12th Midwest Artificial Intelligence and Cognitive Science Conference (MAICS 2001).

DeLoach, S. A., M. F. Wood, and C. H. Sparkman (2001). Multiagent systems engineering. International Journal of Software Engineering and Knowledge Engineering 11(3), 231-258.

Giorgini, P., M. Kolp, J. Mylopoulos, and M. Pistore (2004). The Tropos Methodology: An Overview. Kluwer Academic Publishers.

Giunchiglia, F., J. Mylopoulos, and A. Perini (2002). The tropos software development methodology: Processes, models and diagrams. In Proceedings of the third international workshop on Agent-Oriented Software Engineering.

Grimm, V., U. Berger, F. Bastiansen, S. Eliassen, V. Ginot, J. Giske, J. Goss-Custard, T. Grand, S. K. Heinz, and G. Huse (2006). A standard protocol for describing individual-based and agent-based models. Ecological Modelling 198(1-2), 115-126.

Grimm, V., U. Berger, D. L. DeAngelis, J. G. Polhill, J. Giske, and S. F. Railsback (2010). The ODD protocol: A review and first update. Ecological Modelling 221(23), 2760-2768. 
C. G. Ralha, C. G. Abreu, C. G. C. Coelho and B. Macchiavello, The MASE design experience

Helbing, D. (2012). Social Self-Organization. Springer-Verlag Berlin Heidelberg. Book Subtitle: Agent-Based Simulations and Experiments to Study Emergent Social Behavior, Series Title: Understanding Complex Systems, ISBN: 978-3-642-24003-4 (Print), 978-3-642-24004-1 (Online).

Klink, C. A. and A. G. Moreira (2002). Past and Current Human Occupation, and Land Use. In R. J. Marquis and P. S. Oliveira (Eds.), The Cerrados of Brazil: Ecology and Natural History of a Neotropical Savanna (1st ed.)., Chapter 5, pp. 69-88. New York: Columbia University Press.

May, R. and A. McLean (2007). Theoretical ecology: principles and applications, 3rd edition. Oxford University Press, USA.

McAlpine, C. A., A. Etter, P. M. Fearnside, L. Seabrook, and W. F. Laurance (2009). Increasing world consumption of beef as a driver of regional and global change: A call for policy action based on evidence from Queensland (Australia), Colombia and Brazil. Global Environmental Change 19(1), 21-33.

Oliveira, R. J. M. P. S. (2002). The Cerrados of Brazil: Ecology and Natural History of a Neotropical Savanna (1st ed.). New York: Columbia University Press.

Padgham, L. and M. Winikoff (2002a). Prometheus: a methodology for developing intelligent agents. In Proceedings of the 1st international joint conference on Autonomous agents and multiagent systems: part 1, AAMAS '02, New York, NY, USA, pp. 37-38. ACM.

Padgham, L. and M. Winikoff (2002b). Prometheus: A pragmatic methodology for engineering intelligent agents. In Proceedings of the OOPSLA 2002 - Workshop on Agent-Oriented Methodologies, pp. 97-108.

Padgham, L. and M. Winikoff (2003). Prometheus: A methodology for developing intelligent agents. In F. Giunchiglia, J. Odell, and G. Wei (Eds.), Agent-Oriented Software Engineering III, Volume 2585 of Lecture Notes in Computer Science, pp. 174-185. Springer Berlin Heidelberg.

Parker, D. C., S. M. Manson, M. A. Janssen, M. J. Hoffmann, and P. Deadman (2003). Multi-agent systems for the simulation of land-use and land-cover change: a review. Annals of the Association of American Geographers 93(2), 314-337.

Pontius, R. G., W. Boersma, J.-C. Castella, K. Clarke, T. de Nijs, C. Dietzel, Z. Duan, E. Fotsing, N. Goldstein, K. Kok, E. Koomen, C. D. Lippitt, W. McConnell, A. M. Sood, B. Pijanowski, S. Pithadia, S. Sweeney, T. N. Trung, A. T. Veldkamp, and P. H. Verburg (2008). Comparing the input, output, and validation maps for several models of land change. Annals of Regional Science 42, 11-37.

Railsback, S. F., S. L. Lytinen, and S. K. Jackson (2006). Agent-based simulation platforms: Review and development recommendations. Simulation 82(9), 609-623.

Ralha, C. G., C. G. Abreu, C. G. C. Coelho, A. Zaghetto, B. Macchiavello, and R. B. Machado (2013). A multi-agent model system for land-use change simulation. Environmental Modelling \& Software 42(0), $30-46$.

Rumbaugh, J., I. Jacobson, and G. Booch (2004). Unified Modeling Language Reference Manual, The (2nd Edition). Pearson Higher Education.

Smajgl, A., D. G. Brown, D. Valbuena, and M. G. A. Huigen (2011). Empirical characterisation of agent behaviours in socio-ecological systems. Environ. Model. Softw. 26, 837-844.

Wagner, G. (2003). The agent-object-relationship metamodel: towards a unified view of state and behavior. Information Systems 28(5), 475-504.

Wagner, G. (2004). AOR modelling and simulation: Towards a general architecture for agent-based discrete event simulation. In 5th International Bi-Conference Workshop on Agent-Oriented Information Systems, Volume 3030 of LNCS, pp. 174-188. Springer.

Wagner, G. and I.-M. Diaconescu (2009). Aor-simulation.org: cognitive agent simulation. In C. Sierra, C. Castelfranchi, K. S. Decker, and J. S. Sichman (Eds.), 8th International Joint Conference on Autonomous Agents and Multiagent Systems (AAMAS 2009), Budapest, Hungary, May 10-15, 2009, Volume 2, pp. 14051406. IFAAMAS.

Wooldridge, M., N. R. Jennings, and D. Kinny (2000). The gaia methodology for agent-oriented analysis and design. Autonomous Agents and Multi-Agent Systems 3(3), 285-312. 